\title{
Evaluación de factores histológicos pronósticos de sobrevida en melanoma maligno cutáneo, seguimiento de 13 años*
}

\author{
Drs. ENRIQUE BELLOLIO J. ${ }^{1}$, ALVARO SAN MARTÍN L. ${ }^{2}$, \\ LUIS QUIÑINIIR S. ${ }^{2}$, JUAN ORELLANA C. ${ }^{3}$, OSCAR TAPIA E. ${ }^{,}$, \\ PATRICIO RIFO L. ${ }^{4}$, JUAN CARLOS ROA S. ${ }^{1}$, MIGUEL VILLASECA H ${ }^{1}$.
}

1 Departamento Anatomía Patológica y Scientifical and Technological Bioresources Nucleus (BIOREN).

Internos carrera de Medicina.

3 Centro de Excelencia, Capacitación, Gestión e Investigación para la Salud Basada en Evidencias (CIGES).

4 Dermatólogo, Departamento de Especialidades.

Facultad de Medicina, Universidad de la Frontera, Temuco, Chile.

\begin{abstract}
\section{Prognostic factors for survival of malignant melanomas}

Background: The incidence of malignant melanoma is increasing steadily. Aim: To study pathological prognostic factors in malignant melanomas. Material and Methods: Review of pathological records of cutaneous malignant melanomas diagnosed between 1995 and 2008. Medical records of patients were reviewed and those with distant metastases at the moment of surgery were excluded. Follow up was performed contacting patients and reviewing death records at the Chilean National Identification Registry. The cause of death was classified as related to the melanoma or unrelated. Results: One hundred sixty two records, corresponding to patients aged from 13 to 93 years (51\% women), were included in the study. Twenty nine percent of patients died during the follow up period that ranged from 1 to 127 months. Five and ten year's survival was 71 and $60 \%$ respectively. A multivariable Cox analysis demonstrated that only the TNM classification of the primary tumor, which is categorized using the Breslow maximal depth, had prognostic significance. Compared to women, men had a 1.97 higher relative risk of dying due to the melanoma. Conclusions: TNM classification of melanomas is the most important prognostic factor for survival.
\end{abstract}

Key words: Melanoma, survival, TNM classification.

\section{Resumen}

Introducción: El Melanoma Maligno (MM) de la piel ha aumentado su incidencia en 5\% anual, se estima que 1 de cada 55 hombres y 1 de cada 77 mujeres desarrollará la enfermedad durante su vida. Método y Diseño: Estudio de cohorte retrospectivo de seguimiento de casos de melanoma maligno primario de la piel diagnosticados en la Unidad de Anatomía Patológica del Hospital Dr. Hernán Henríquez Aravena de Temuco. Se incluyeron 162 casos de MM primario de piel. El período de seguimiento fue de 1 a 164 meses. El análisis

\footnotetext{
*Recibido el 4 de Enero de 2010 y aceptado para publicación el 10 de Marzo de 2010.

Correspondencia: Dr. Enrique Bellolio J. Manuel Montt 112. Código postal 478-1176. Temuco, Chile. E-mail: ebellolio@gmail.com
} 
estadístico se realizó según método de Kaplan-Meier para la descripción de sobrevida según las variables de interés, utilizando la prueba no paramétrica Log-rank, modelo univariado y multivariado de Cox. Resultados: Se incluyeron 162 casos en la cohorte de estudio. El 28,7\% de los pacientes falleció producto del melanoma durante el tiempo de observación (rango 1 a 127 meses, mediana 23 meses). La sobrevida general de la cohorte a 5 y 10 años fue de $71 \%$ y $60 \%$ respectivamente. El análisis multivariado de Cox demostró que sólo la clasificación del tumor primario según TNM es significativa. Los hombres tienen un riesgo relativo de 1,97 para morir por MM. La edad y distribución topográfica no resultaron ser variables pronósticas. Conclusión: En nuestro estudio se confirman los indicadores histológicos de pronóstico de sobrevida en población de la región de La Araucanía, los factores de mayor importancia son el espesor máximo según Breslow, presencia de úlcera, tipo histológico y nivel de infiltración de Clark.

Palabras clave: Melanoma, patología, epidemiología.

\section{Introducción}

El Melanoma Maligno (MM) primario de la piel es una neoplasia derivada de los melanocitos, que ha aumentado su incidencia en todos los países del mundo en promedio 5\% anual. A pesar de que el MM es la causa más común de muerte por cáncer de la piel, sólo representa el 4\% de los cánceres cutáneos ${ }^{1}$. Durante el año 2002 aproximadamente 79.000 hombres y 81.000 mujeres fueron diagnosticados con melanoma en el mundo y de ellos aproximadamente el $80 \%$ fueron en personas de piel blanca, con una mortalidad de 22.000 hombres y 19.000 mujeres $^{2,3}$. En Estados Unidos se ha reportado un incremento de hasta $600 \%$ en el diagnóstico de melanoma cutáneo desde 1950 hasta el año 2000, con una incidencia de 2,9 por 100.000 habitantes para el año 2002. En el año 2005 el número estimado de casos nuevos diagnosticados fue de 59,58 en este país y de estos 7.700 podrían morir por esta enfermedad ${ }^{4}$. A pesar de su baja prevalencia, se estima que uno de cada 55 hombres y una de cada 77 mujeres desarrollará esta neoplasia durante su vida ${ }^{5}$. Por otra parte, en Chile se ha experimentado un aumento en $105 \%$ de su incidencia entre los años 1992 y $1998^{6}$; la mortalidad se ha incrementado en $12 \%$ entre los años 1988 y 1998 en los hospitales de Santiago?.

En cuanto a los factores de riesgo, el principal factor ambiental es la exposición a radiación ultravioleta, particularmente intermitente antes de los 20 años, así como también la residencia en mayores latitudes ${ }^{8}$, en combinación con factores endógenos como tipos de piel I y II, inmunodeficiencia y predisposición genética9 ${ }^{9}$ Por otra parte, el número de nevos comunes y nevos atípicos también ha mostrado ser un importante factor de riesgo para desarrollar $\mathrm{MM}^{10}$.

Con respecto a la distribución topográfica del MM, según datos europeos, es común en los miembros inferiores, pero la presentación ha variado en los últimos años, aumentando las localizaciones en el tronco, sobre todo en el varón, así como las formas pequeñas y poco invasoras, debido quizás a la mayor precocidad con que se efectúa el diagnósti$\mathrm{co}^{11}$. En casuísticas nacionales se ha visto una mayor incidencia en cabeza, seguido de miembros inferiores $^{12}$. Sobre el valor pronóstico de la localización del MM en la sobrevida a los 5 y 10 años, algunos han planteado que los de cabeza y cuello mueren 1,84 veces más rápido comparado con los $\mathrm{MM}$ de otras localizaciones ${ }^{13,14}$.

Respecto al género, se ha asociado a una peor sobrevida para los hombres con cifras a los 5 y 10 años de $69 \%$ y $61 \%$, comparado con las mujeres con un $82 \%$ y $75 \%$, respectivamente ${ }^{15}$.

El espesor máximo según Breslow ${ }^{16}$ y la presencia de úlcera, son factores pronósticos independientes de sobrevida ${ }^{17}$. Estos factores adquieren utilidad según el estado en que se presenta el melanoma al momento del diagnóstico, siendo el espesor máximo según Breslow, la presencia de ulceración y el nivel de invasión de Clark $^{18}$ los factores pronósticos más importantes en MM con espesor menor a $1 \mathrm{~mm}$ para el sistema de etapificación del American Joint Committee on Cancer ${ }^{19}$. El número de linfonodos metastáticos, la presencia de metástasis intralinfática y la ulceración son importantes en melanomas en etapa III, mientras que en melanomas metastáticos los factores más importantes son el sitio de metástasis y la elevación de isoenzima deshidrogenasa láctica (LDH) sérica ${ }^{20}$.

El objetivo del estudio es cuantificar y determinar qué factores histológicos son de relevancia pronóstica en la sobrevida de pacientes diagnosticados con MM en el Hospital Hernán Henríquez Aravena de Temuco entre los años 1995 y 2008.

\section{Material y Método}

Los pacientes fueron seleccionados de la base de datos informatizada de la Unidad de Anatomía Patológica del Hospital Dr. Hernán Henríquez Aravena de Temuco. Se incluyó la totalidad de casos de MM 
cutáneo en el período comprendido entre el 01 de enero de 1995 y el 31 de octubre de 2008. Se revisaron los informes de biopsias, fichas clínicas y láminas histológicas para obtener los datos de las variables de interés. Se excluyeron los pacientes que presentaban metástasis a distancia al momento de la cirugía. El seguimiento se realizó con controles de los pacientes y en base de datos de registro nacional de identificación, objetivando la causa de muerte, diferenciando las debidas a MM y otras causas no relacionadas a la enfermedad en estudio.

El análisis estadístico se llevó a cabo utilizando el programa Stata 10. Se realizó análisis de sobrevida según método de Kaplan-Meier para la descripción de la sobrevida según las variables de interés, se utilizó la prueba no paramétrica Log-rank para comparar inferencialmente la sobrevida de grupos y el modelo univariado y multivariado de Cox para el estudio conjunto de las variables de interés sobre la sobrevida de los pacientes ${ }^{21,22}$.

\section{Resultados}

Del total de 165 casos de la población de referencia, 162 tenían toda la información necesaria para este estudio. La mediana de seguimiento fue de 61 meses, con un rango entre 1 y 164 meses. La distribución general de género en la cohorte fue de $51 \%$ para mujeres y $49 \%$ para hombres, no existiendo diferencia estadística en la distribución. La mediana y promedio para edad fue 63 y 60,2 años respectivamente, con un rango de edad entre 13 y 93 años. Se realizó análisis estratificado por quintiles de edad no encontrando diferencia estadística.

El $28,7 \%$ de los pacientes falleció producto del MM durante el período de seguimiento. La tasa densidad de incidencia de muerte por MM fue de 0,0562 fallecimientos por año/persona de observación. En la Tabla 1 se detallan las características generales, y en la Figura 1 la sobrevida general de la cohorte.

En la Tabla 2 se muestran indicado- res de asociación cruda expresados como densidad de incidencia, Razón de Hazard y sobrevida a 5 y 10 años, para género, edad mayor y menor de 45 años, presencia de úlcera, espesor máximo según Breslow (mm), nivel de Clark, tipo histológico y clasificación del tumor primario según $\mathrm{AJCC}^{19}$, generando grupos que discriminan el riesgo. La edad es la única variable que no tiene mayor riesgo asociado de morir en los dos grupos etáreos comparados, siendo el resto de las variables estadísticamente significativas $(\mathrm{p}<0,05)$. Destaca el riesgo de casi 4 veces mayor para aquellos pacientes con MM de más de $3 \mathrm{~mm}$ de espesor. También se puede ver que los pacientes clasificados como pT4 tienen un

Tabla 1. Características generales de la cohorte

\begin{tabular}{llc}
\hline Características & & $\mathbf{n}=\mathbf{1 6 2}(\mathbf{\%})$ \\
\hline Género & Femenino & $80(49)$ \\
& Masculino & $82(51)$ \\
Edad (años) & Media & 60,1 \\
& Rango (mín-máx) & $13-93$ \\
Localización del & Cabeza y cuello & $44(26,8)$ \\
melanoma & Tronco & $15(9,1)$ \\
& Miembro superior & $19(11,6)$ \\
& Miembro inferior & $76(46,3)$ \\
& Otras localizaciones & $10(6,1)$ \\
Úlcera & Presente & $47(28,7)$ \\
Estado al fin del & Ausente & $117(71,3)$ \\
seguimiento & Vivos & $93(56,7)$ \\
& Muertos por melanoma & $47(28,7)$ \\
& Muertos por otra causa & $24(14,6)$ \\
\hline
\end{tabular}

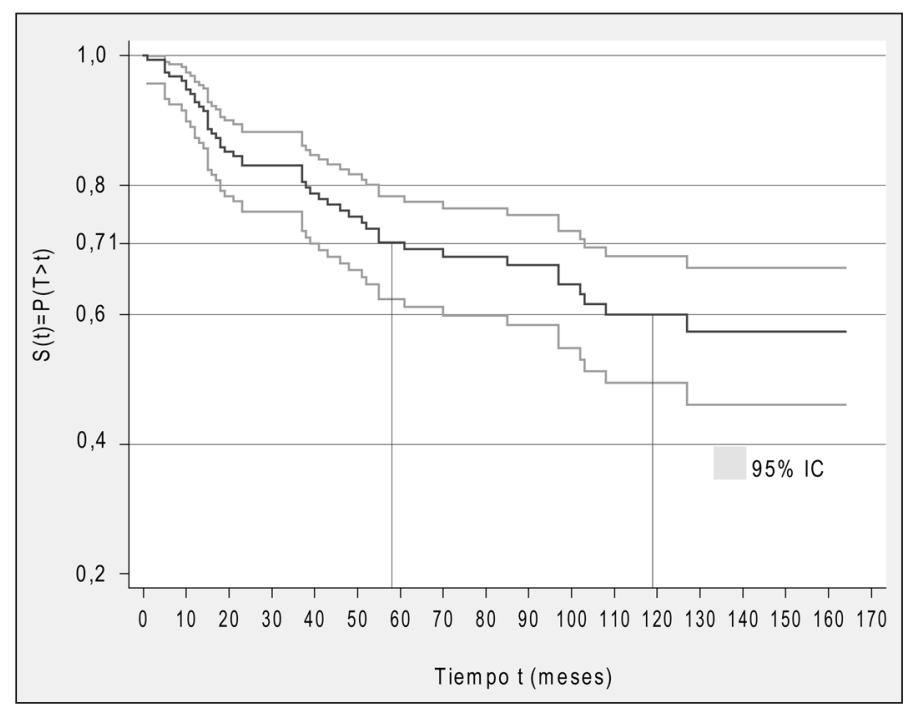

Figura 1. Sobrevida estimada Kapplan-Meier. 
Tabla 2. Densidad de incidencia (DI), Razón de Hazard (RH) y sobrevida a 5 y 10 años

\begin{tabular}{|c|c|c|c|c|c|c|c|c|}
\hline \multirow[t]{3}{*}{ Variables } & & \multirow{3}{*}{$\begin{array}{c}\text { DI } \\
\text { año } \\
\text { persona }\end{array}$} & \multirow{3}{*}{$\underset{*}{\mathbf{R H}}$} & \multirow{3}{*}{$\begin{array}{l}\text { Valor } \\
\text { p * }\end{array}$} & \multicolumn{4}{|c|}{ Porcentaje de sobrevida } \\
\hline & & & & & \multicolumn{2}{|c|}{5 años } & \multicolumn{2}{|c|}{10 años } \\
\hline & & & & & $\hat{\mathbf{S}}$ & IC95\% & $\hat{\mathbf{S}}$ & IC95\% \\
\hline \multirow[t]{2}{*}{ Género } & Femenino & 0,0769 & 1,00 & - & 63,2 & $50,1-73,8$ & 51,0 & $35,9-64,3$ \\
\hline & Masculino & 0,0381 & 1,98 & 0,024 & 79,4 & $67,0-87,6$ & 69,2 & $54,1-80,1$ \\
\hline \multirow[t]{2}{*}{ Edad (años) } & 45 y menos & 0,0324 & 1,00 & - & 82,3 & $62,4-92,3$ & 72,9 & $50,7-86,3$ \\
\hline & $>45$ & 0,0661 & 1,86 & 0,111 & 68,1 & $57,9-76,4$ & 55,9 & $43,3-66,8$ \\
\hline \multirow[t]{2}{*}{ Úlcera } & Ausente & 0,0435 & 1,00 & - & 77,0 & $66,9-84,4$ & 69,2 & $57,0-78,5$ \\
\hline & Presente & 0,0928 & 2,06 & 0,014 & 57,1 & $39,5-71,3$ & 39,2 & $21,2-56,8$ \\
\hline \multirow{3}{*}{$\begin{array}{l}\text { Espesor máximo } \\
\text { según Breslow (mm) }\end{array}$} & {$[0,0-2,0]$} & 0,0285 & 1,00 & - & 86,7 & $77,1-92,5$ & 74,1 & $60,7-83,6$ \\
\hline & {$[3,0-6,0]$} & 0,1198 & 3,77 & 0,000 & 48,9 & $27,6-67,2$ & 39,1 & $16,6-61,1$ \\
\hline & {$[7,0-30]$} & 0,1299 & 4,29 & 0,000 & 41,8 & $21,0-61,4$ & 33,4 & $13,3-55,2$ \\
\hline \multirow[t]{3}{*}{ Nivel de Clark } & 1 ó 2 & 0,0074 & 1,00 & - & 95,2 & $82,2-98,8$ & 95,2 & $82,2-98,8$ \\
\hline & 3 ó 4 & 0,0617 & 8,15 & 0,004 & 71,0 & $58,0-80,6$ & 54,9 & $39,3-68,0$ \\
\hline & 5 & 0,1312 & 17,1 & 0,000 & 42,4 & $24,2-59,6$ & 31,8 & $14,7-50,5$ \\
\hline \multirow[t]{3}{*}{ Tipo histológico } & $\begin{array}{l}\text { Ext. superficial / } \\
\text { Léntigo maligno }\end{array}$ & 0,0221 & 1,00 & - & 88,3 & $75,4-94,7$ & 78,0 & $60,6-88,4$ \\
\hline & Nodular & 0,0911 & 4,03 & 0,000 & 58,1 & $43,1-70,4$ & 51,0 & $34,8-65,0$ \\
\hline & Acral lentiginoso & 0,0842 & 3,63 & 0,003 & 59,6 & $37,4-76,4$ & 38,7 & $16,6-60,5$ \\
\hline \multirow{3}{*}{$\begin{array}{l}\text { Clasificación tumor } \\
\text { primario (AJC) }\end{array}$} & T 1 & 0,0092 & 1,00 & - & 95,6 & $83,2-98,9$ & 90,3 & $69,8-97,1$ \\
\hline & T 2 ó 3 & 0,0488 & 5,24 & 0,010 & 74,2 & $56,8-85,4$ & 66,4 & $47,4-79,8$ \\
\hline & T 4 & 0,1265 & 13,12 & 0,000 & 47,3 & $32,5-60,8$ & 29,9 & $15,8-45,4$ \\
\hline
\end{tabular}

*Estimación de la Razón de Hazard y valor p usando el modelo de Hazard proporcional de Cox.

riesgo de morir hasta 13 veces mayor al compararlo con los pT1. Las Figuras 2, 3, 4 y 5 muestran la estimación de la sobrevida por Kaplan-Meier según cada variable de interés. En la Tabla 3 se muestran los resultados del análisis univariado de Cox: género, en el que los hombres tienen un riesgo de morir 2 veces mayor que las mujeres; la presencia de úlcera se traduce en que los pacientes tienen un riesgo 2 veces mayor que los que no la presentan; nivel de Clark, respecto del nivel 1 ó 2 , el nivel 3 ó 4 y nivel 5, tienen un riesgo de morir de 7,5 y 14 veces mayor respectivamente; tipo histológico, respecto de los tipo Extensión superficial/Lentigo Maligno, el tipo nodular y acral lentiginoso muestran un riesgo de morir de 4 y 3,6 veces mayor, respectivamente; la clasificación del tumor primario según

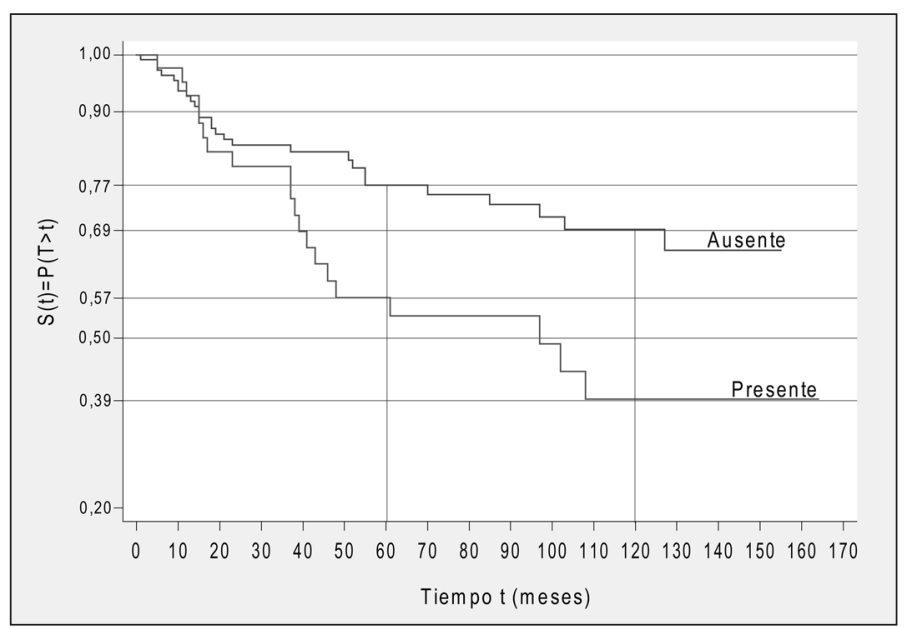

Figura 2. Sobrevida estimada Kapplan-Meier según presencia de úlcera. 
AJCC (pT), respecto de T1, los grupos T2 o T3 y T4, muestran respectivamente 5 y 13 veces mayor riesgo de morir.

Con el fin de estudiar la asociación entre los distintos factores explicativos sobre la sobrevida, se procede a realizar el análisis multivariado de Cox. De este último se obtuvo que la clasificación del tumor pT, según AJCC, es la única variable con significancia pronóstico independiente.

La ubicación topográfica del MM resultó ser de mayor a menor frecuencia en extremidad inferior $46 \%$, cabeza y cuello $26 \%$, extremidad superior $12 \%$, tronco $11 \%$ y otras ubicaciones $6 \%$, sin significancia pronóstico $(\mathrm{p}=0,079)$.

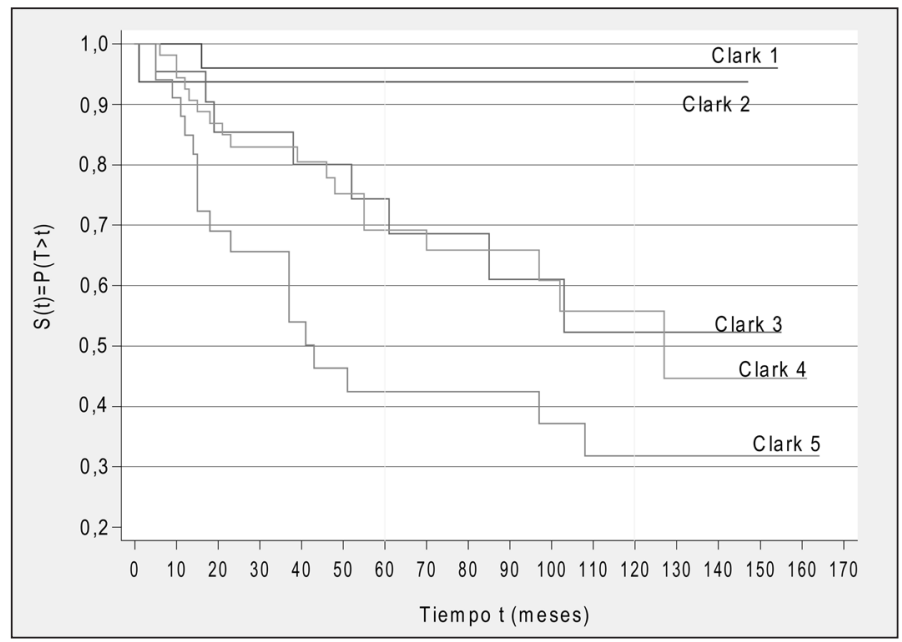

Figura 3. Sobrevida observada según grupos de nivel de Clark.

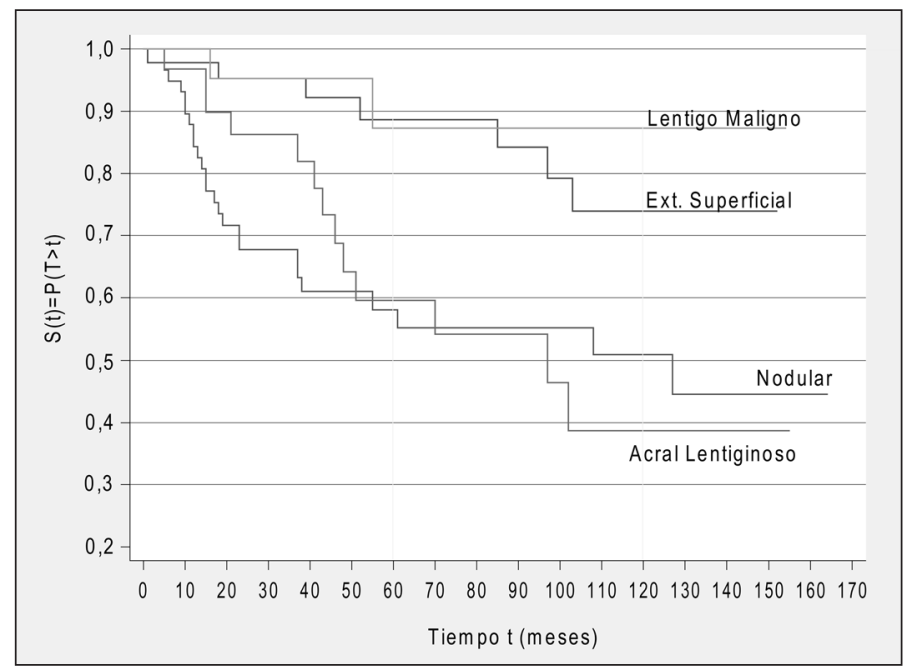

Figura 4. Sobrevida observada según tipo histológico.
La disección ganglionar linfática regional se realizó en 55 pacientes, lo que representa el $34 \%$ de la cohorte. Excluyendo las lesiones menores de $1 \mathrm{~mm}$ de espesor, el porcentaje de disección ganglionar llega a 42,6\%. El análisis demostró que los pacientes con vaciamiento ganglionar presentan un riesgo de morir 2,4 veces mayor que los que no se realizó $(\mathrm{p}<0,01)$. $\mathrm{Al}$ evaluar los que tenían metástasis de melanoma se encontró que estos tienen un riesgo de morir 3,1 veces mayor que los que no tienen metástasis $(\mathrm{p}<0,01)$

En 37 pacientes se indicó tratamiento adyuvante, con sólo quimioterapia en 26 casos, sólo radioterapia en 5 casos y terapia combinada en 6 casos. Su distribución según el pT fue: 3 en pT 1 , ninguno en pT2, 9 en pT3 y 25 en pT4. Los pacientes que recibieron tratamiento adyuvante tienen un riesgo de morir 2,8 veces mayor que los que no lo recibieron $(\mathrm{p}<0,01)$.

\section{Discusión}

De los resultados obtenidos, podemos señalar que son similares a los reportados por Balch et $\mathrm{al}^{17}$, trabajo que presenta la mayor cohorte descrita, con 17.600 casos de 13 instituciones norteamericanas. Lo anterior, permite validar la clasificación TNM propuesta por AJCC en nuestra población de estudio de la Región de La Araucanía. Desde el punto de vista histológico, el grado de Tumor (pT), se clasifica utilizando el espesor máximo según Breslow expresado en $\mathrm{mm}$, situación ampliamente difundida en la literatura. La presencia de úlcera ha sido un factor estudiado, que ha demostrado ser de peor pronóstico en los casos en que está presente, situación similar a la encontrada en nuestro estudio ${ }^{23-26}$.

La clasificación clinicopatológica de MM en extensión superficial, lentigo maligno, nodular y acral lentiginoso ${ }^{27}$, demuestra un valor pronóstico en el análisis univariado de Cox, con un riesgo relativo de morir de hasta casi 3 y 4 veces más para los nodulares y los de tipo acral lentiginoso, respectivamente; situación que en la literatura 


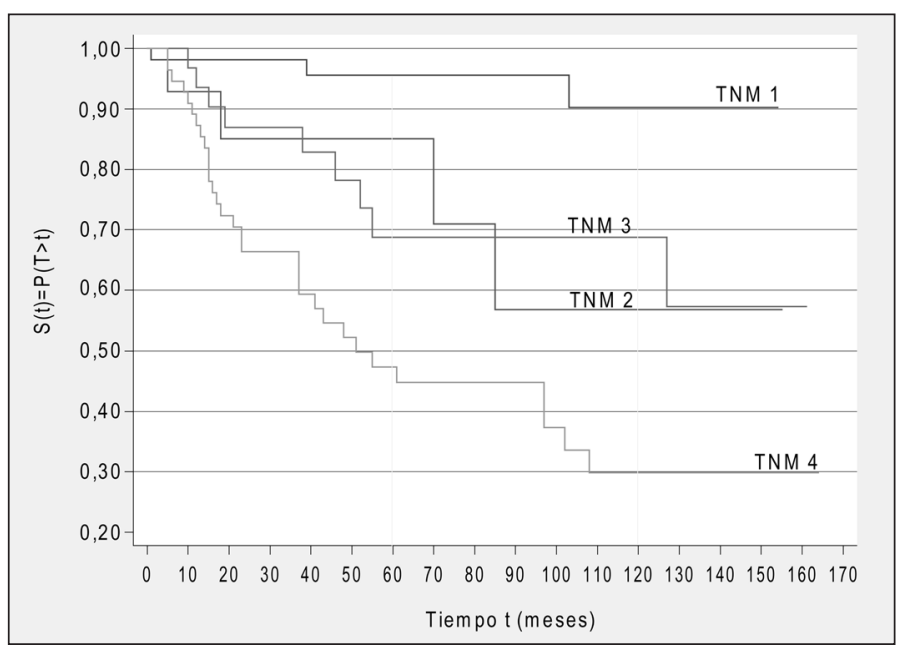

Figura 5. Sobrevida observada según clasificación del tumor primario por AJCC.

Tabla 3. Estimación de la Razón de Hazard por modelo de Cox para cada una de las variables de interés controlando por sexo y edad $>45$ años

\begin{tabular}{llccc}
\hline Variables & & \multicolumn{2}{c}{ HR } & Valor p \\
& & HR & IC 95\% & \\
\hline Género & Femenino & 1,00 & - & - \\
Edad (años) & Masculino & 1,97 & $1,09-3,58$ & 0,026 \\
& 45 y menos & 1,00 & - & - \\
Presencia de úlcera & 45 & 1,85 & $0,86-3,99$ & 0,116 \\
& Ausente & 1,00 & - & - \\
Nivel de Clark & Presente & 1,92 & $1,07-3,44$ & 0,028 \\
& 1 ó 2 & 1,00 & - & - \\
\multirow{2}{*}{ Tipo histológico } & ó 4 & 7,54 & $1,78-31,88$ & 0,006 \\
& 5 & 14,37 & $3,30-62,56$ & 0,000 \\
& Ext. superficial /Léntigo maligno & 1,00 & - & - \\
Clasificación TNM & Nodular & 3,75 & $1,73-8,10$ & 0,001 \\
& Acral lentiginoso & 2,95 & $1,23-7,02$ & 0,015 \\
& TNM 1 & 1,00 & - & - \\
& TNM 2 ó 3 & 4,65 & $1,32-16,43$ & 0,017 \\
& TNM 4 & 11,71 & $3,53-38,79$ & 0,000 \\
\hline
\end{tabular}

sobre el tema ha dejado de ser mencionada, dando mayor importancia al espesor máximo de Breslow y nivel de infiltración de Clark. Creemos que el tipo histológico tiene cierta correlación con el espesor de Breslow, siendo en nuestra cohorte los de mayor espesor los melanomas nodulares con $7,3 \mathrm{~mm}$ de espesor promedio, seguidos por los de tipo acral lentiginoso con 4,2 $\mathrm{mm}$ de espesor promedio. Esto podría explicar en parte el mayor riesgo de estos dos subtipos clinicopatológicos de MM.
La ubicación topográfica confirma una distribución similar a la descrita en otros trabajos revisados, pero no mostró que existiera una ubicación con mayor riesgo de morir ${ }^{12,15}$.

Se realizó disección ganglionar linfática regional al 34\% del total de pacientes. Este porcentaje llega al $38 \%$ y $57 \%$ para los pacientes con pT3 y pT4 respectivamente.

Hay estudios que evalúan la utilización de otros factores pronósticos en MM, se han utilizado técni- 
cas como inmunohistoquímica e inmunofluorescencia con hibridación in-situ, del inglés Fluorescence In Situ Hybridization (FISH) para su identificación y determinación. Destaca la detección la proteína G reguladora de la señal 1 RGS1 ("regulated G protein signaling 1") mediante inmunohistoquímica, en la que un aumento en la expresión de esta proteína se ha asociado a un peor pronóstico, lo que también se asoció a estadios más avanzados y fenotipo más agresivo de la enfermedad ${ }^{28}$. Un aumento de la expresión de ciclooxigenasa isoenzima-2 (COX-2) en MM cutáneo se ha asociado a niveles de Clark elevados y a mayor probabilidad de recurrencia de la enfermedad ${ }^{29}$. La expresión de c-kit (CD117), un receptor transmembrana de tirosina-kinasa, se ha asociado a hiperpigmentación y su disminución a fase de crecimiento vertical en $\mathrm{MM}^{30}$.

Respecto de las alteraciones citogenéticas que se encuentran en MM tienen relación con alteraciones de las vías de los oncogenes BRAF y NRAS, en las que se han comprobado mediante FISH aberraciones cromosómicas con pérdida o aumento de copias de cromosomas. Esto se ha comprobado comparando MM con lesiones melanocíticas névicas, lo que ha permitido en casos de difícil diagnóstico hacer una diferencia utilizando esta técnica, lo que siempre debe ser evaluado en conjunto con los antecedentes clínicos e histopatológicos de cada caso ${ }^{31}$.

Como se puede deducir de lo revisado, aún no hay marcadores $\mathrm{u} / \mathrm{o}$ antígenos que permitan predecir de mejor manera la sobrevida de pacientes con MM que los descritos con la histopatología clásica. El avance alcanzado en citogenética por FISH tendrá un rol preponderante en el futuro en esta patología.

Para concluir, cabe señalar que los factores de mayor importancia son el espesor máximo según Breslow, presencia o ausencia de úlcera, tipo histológico y nivel de infiltración de Clark. Esta información debe estar disponible en el reporte de anatomía patológica de melanoma maligno de la piel, lo que permite al médico tratante y paciente tener una aproximación válida y demostrada respecto de la sobrevida $^{32}$.

\section{Referencias}

1. Balch CM, Reintgen DS, Kirkwood JM, Houghton A, Peters L, Ang KK, et al. Cancer: Principles and Practice of Oncology. $5^{\text {th }}$ Edn. Lippincott-Raven Publihers, Philadelphia, 1997 Ch.41.2.

2. Ferlay J, Bray FI, Pisani P, Parkin DM. Globocan 2000: Cancer incidence, mortality, and prevalence worldwide. 1ed. Lyon: IARC Press; 2001.

3. Leboit PH, Burg G, Weedon D, Sarasin A. Pathology and Genetics of Skin Tumours; International Agency for Research on Cancer. Chapter 2: 49-120. IARCPress
2006.

4. Homsi J, Kashani-Sabet M, Messing JL, Daud A. Cutaneous melanoma: prognostic factors. Cancer Control 2005; 12: 223-229.

5. Jemal A, Siegel R, Ward E, Murray T, Xu J, Smigal C, et al. Cancer statistics, 2006. CA Cancer J Clin 2006; 56: 106-130.

6. Zemelman V, Roa J, Díaz C. Aumento de la incidencia del cáncer cutáneo en hospitales públicos de la Región Metropolitana (1992-1998). Revista Chilena de Dermatología 2001; 17: 180-185.

7. Zemelman V, Garmendia ML, Kirschbaum A. Malignant melanoma mortality in Chile (1988-1998). Int J Dermatol 2002; 41: 99-103.

8. Elwood JM, Diffey BL. A consideration of ambient solar ultraviolet radiation in the interpretation of studies of the etiology of melanoma. Melanoma Res 1993; 3 : 113-122.

9. Armstrong Kricker A. How much melanoma is caused by sun exposure? Melanoma Res 1993; 3: 395-401.

10. Gandini S, Sera F, Catttaruzza Ms, Pasquini P, Abeni $\mathrm{D}$, Boyle P, et al. Meta-analysis of risk factors for cutaneous melanoma: Common and atypical naevi. Eur J Cancer 2005; 41: 28-44.

11. Meiriño R, Martínez E, Marcos M, Villafranca E, Domínguez MA, Illarramendi JJ, y cols. Factores pronósticos en el melanoma maligno cutáneo. An Sist Sanit Navarr 2001; 24: 167-172.

12. Benedetto J, Balestrini C, Silva F, Molgo M, González S. Tipos histológicos y variables pronósticas en 596 casos de melanoma maligno de la piel, entre 1976 y 2001. Revista Chilena de Dermatología 2003; 19: 94-99.

13. Gillgren P, Månsson-Brahme E, Frisell J, Johansson H, Larsson O, Ringborg U. A prospective population-based study of cutaneous malignant melanoma of the head and neck. Laryngoscope 2000; 110: 1498-1504.

14. Lachiewicz M, Berwick M, Wiggins ChL, Thomas NE. Survival Differences Between Patients With Scalp or Neck Melanoma and Those With Melanoma of Other Sites in the Surveillance, Epidemiology, and End Results (SEER) Program. Arch Dermatol. 2008; 144: 515521.

15. MacKie RM, Hole D, Hunter JA, Rankin R, Evans A, McLaren K, et al. Cutaneous malignant melanoma in Scotland: incidence, survival, and mortality, 1979-94. The Scottish Melanoma Group. BMJ 1997; 315: 11171121.

16. Breslow A. Thickness, cross-sectional areas and depth of invasion in the prognosis of cutaneous melanoma. Ann Surg 1970; 172: 902-908.

17. Balch ChM, Soong SJ, Gershenwald JE, Thompson JF, Reintgen DS, Cascinelli N, et al. Prognostic Factors Analysis of 17,600 Melanoma Patients: Validation of the American Joint Committee on Cancer Melanoma Staging System. Journal of Clinical Oncology 2001; 19: 3622-3634. 
18. Clark WH Jr, From L, Bernardino EA, Mihm MC. The histogenesis and biologic behavior of primary human malignant melanomas of the skin. Cancer Res 1969; 29: 705-727.

19. AJCC Cancer Staging Atlas. Part V; Chapter 24: 207216. (C2006 Springer

20. Balch ChM, Soong SJ, Atkins MB, Buzaid AC, Cascinelli N, Coit DG, et al. An Evidence-based Staging System for Cutaneous Melanoma. CA Cancer J Clin 2004; 54: 131-149.

21. Dupont W. Statistical Modeling for Biomedical Researchers. (C2002 Cambridge University Press.

22. Hamilton LC. Statistics with Stata. (C2006 Duxbury, Thomson Brooks/Cole.

23. Balch CM. Cutaneous melanoma: prognosis and treatment results worldwide. Semin Surg Oncol 1992; 8: 400-414.

24. Cochran AJ, Elashoff D, Morton DL, Elashoff R. Individualized prognosis for melanoma patients. Hum Pathol 2000; 31: 327-331.

25. S. Retsasa, K. Henryb, M.Q. Mohammeda, K. MacRaec. Prognostic factors of cutaneous melanoma and a new staging system proposed by the American Joint Committee on Cancer (AJCC): validation in a cohort of 1284patients. European Journal of Cancer 2002; 38: 511-516.

26. Thomas K. Eigentler, Petra G. Buettner, Ulrike Leiter, and Claus Garbe. Impact of Ulceration in Stages I to III Cutaneous Melanoma As Staged by the Ameri- can Joint Committee on Cancer Staging System: An Analysis of the German Central Malignant Melanoma Registry. Journal of Surgical Oncology 2004; 21 : 4376-4383.

27. Barnhill RL. Malignant melanoma: histology. In: Miller SJ, Maloney ME (eds): Cutaneus Oncology: Pathophysioloy, Diagnosis, and Treatment. Cambridge, MA, Blackwell Science, 1996, p 262-277.

28. Rangel J, Nosrati M, Leong S, Haqq C, Miller JR, Sagebiel RW, et al. Novel Role for RGS1 in Melanoma Progression. Am J Surg Pathol 2008; 32: 1207-1212.

29. Meyer S, Vogt T, Landthaler M, Berand A, Reichle A, Bataille F, et al. Cyclooxygenase 2 (COX2) and Peroxisome Proliferator-Activated Receptor Gamma (PPARG) Are Stage-Dependent Prognostic Markers of MalignantMelanoma. PPAR research 2009: 848645. Epub 2009 Jul 20.

30. Wu JM, Álvarez H, García P, Rojas PL, Wong G, Maitra A, et al. Melanoma Hyperpigmentation Is Strongly Associated With KIT Alterations. Am J Dermatopathol 2009; 31: 619-625.

31. Gerami P, Wass A, Mafee M, Fang Y, Pulitzer MP, Busam KJ. Fluorescence In Situ Hybridization (FISH) as an Ancillary Diagnostic Tool in the Diagnosis of Melanoma. Am J Surg Pathol 2009; 33: 1146-1156.

32. Scolyer RA, Thompson JF, Stretch JR, Sharma R, McCarthy M. Pathology of Melanocytic Lesions: New, Controversial, and Clinically Important Issues. Journal of Surgical Oncology 2004; 86: 200-211. 